\title{
PREDIÇÃO DA TOLERÂNCIA AO DESSECAMENTO DE SEMENTES FLORESTAIS AMAZÔNICAS
}

\author{
Jarde da Silva Freitas ${ }^{1}$ Marilene de Campos Almeida ${ }^{2}$ \\ ${ }^{1}$ Graduado do Curso de Engenharia Florestal da Universidade Federal do Acre - Rio \\ Branco, Acre, Brasil. (freitasxapuri@hotmail.com) \\ ${ }^{2}$ Pesquisadora Doutora do Laboratório de Sementes Florestais do Parque \\ Zoobotânico da Universidade Federal do Acre- Rio Branco, Acre, Brasil. \\ marilenepz@yahoo.com.br
}

\section{Recebido em: 08/04/2016 - Aprovado em: 30/05/2016 - Publicado em: 20/06/2016 DOI: 10.18677/Enciclopedia_Biosfera_2016_086}

\begin{abstract}
RESUMO
O objetivo deste trabalho foi prever a tolerância ao dessecamento de sementes de vinte e quatro espécies amazônicas regionais pelo método "Seed Coat Ratio" SCR. Avaliando-se as seguintes espécies: Handroanthus impetiginosus, Handroanthus serratifolius, Aspidosperma vargasii, Hymenaea courbaril, Bertholletia excelsa, Enterolobium schomburgkii, Guarea sp, Hymenaea stignocarpa, Dipterix odorata, Buchenavia grandis, Tetragastris altissima, Inga sp, Pouteria reticulata, Carapa guianensis, Garcinia macrophylla, Aspidosperma macrocarpon, Dialium guianense, Manilkara inundata, Drypetes sp., Hevea brasiliensis, Mabea anadendra, Abuta grandifolia, Virola surinamensis e Castilla ulei. Para a previsão da tolerância ao dessecamento, foram utilizadas 10 sementes, dissecadas em: endoscarpo+ testa (envoltório) e eixo embrionário + cotilédones e endosperma (semente). O Material foi pesado e levado a temperatura de $105^{\circ} \mathrm{C} \pm 3$, por 24 horas, calculado o peso de massa seca, o SCR e o valor de P de acordo com a equação I. A previsão da tolerância da semente ao dessecamento pelo método SCR mostrou-se eficiente para $42 \%$ das espécies estudadas. O método SCR é prático por não necessitar realizar testes de germinação, mas deve ser utilizado associando-se outros critérios na préclassificação das sementes, uma vez que o método apenas classifica as sementes em ortodoxas e recalcitrantes, não considerando a categoria intermediária ou o nível de recalcitrância das sementes.
\end{abstract}

PALAVRAS-CHAVE: armazenamento; classificação, semente florestal

\section{PREDICTION OF DESICCATION TOLERANCE OF AMAZONIAN FOREST SEEDS}

\begin{abstract}
The objective of this study was to predict the desiccation tolerance of seeds from 12 regional tree species from the Amazon by the "Seed Coat Ratio" - SCR. Seeds from 12 forest species were used in the study: Handroanthus impetiginosus, Handroanthus serratifolius, Aspidosperma vargasii, Hymenaea courbaril, Bertholletia excelsa, Enterolobium schomburgkii, Guarea sp, Hymenaea stignocarpa, Dipterix odorata, Buchenavia grandis, Tetragastris altissima, Inga sp, Pouteria reticulata, Carapa guianensis, Garcinia macrophylla, Aspidosperma macrocarpon, Dialium guianense, Manilkara inundata, Drypetes sp., Hevea brasiliensis, Mabea anadendra, ENCICLOPÉDIA BIOSFERA, Centro Científico Conhecer - Goiânia, v.13 n.23; p.1002 2016
\end{abstract}


Abuta grandifolia, Virola surinamensis e Castilla ulei. For the prediction of tolerance to desiccation, 10 seeds were used, which were dissected in: endocarp + test (wrap) and the embryonic axis + cotyledons and endosperm (seed). The seeds were weighed, calculated the SCR and the calculated value P according to the equation The prediction of tolerance to desiccation of the seed by the SCR method was effective for $42 \%$ of the species. The SCR method, although rapid and practical it does not require germination tests should be used by associating to other criteria in the pre-sorting of the seeds, since the method only classifies the orthodox and recalcitrant seeds in not considering the intermediate category or level of recalcitrance

KEYWORDS: Classification, storage, forest seed

\section{INTRODUÇÃO}

A partir da década de 1990, em razão do enfoque em recuperação e conservação de ecossistemas, ocorreu acentuado interesse em compreender o comportamento de sementes de espécies nativas durante o armazenamento Entretanto, a preservação da qualidade das sementes durante a estocagem requer a integração de vários fatores, como temperatura, umidade relativa, tipo de embalagem e grau de umidade das sementes (BRASIL, 2009). Segundo HONG et al. (1996), para a efetiva conservação de sementes, é necessário o conhecimento prévio do seu comportamento fisiológico durante a secagem e o armazenamento a longo prazo, já que nem todas as sementes são tolerantes à dessecação, exigindo condições especiais de armazenamento (GOMES et al., 2013; LIMA et al., 2014).

A tolerância à dessecação consiste na capacidade dos organismos de recuperar as funções biológicas quando são reidratados após terem sido submetidos à desidratação natural ou artificial (Rego et al., 2013). Esta é uma importante estratégia de adaptação, pois mantém sua viabilidade por um longo período de tempo em condições favoráveis (PELISSARI et al, 2013).

Segundo VINAYACHANDRA \& CHANDRASHEKAR (2011), informações sobre a longevidade das sementes, sensibilidade à dessecação e ao congelamento são pré-requisitos para a conservação de espécies de plantas que, geralmente, produzem sementes não-ortodoxas. A longevidade das sementes e o comportamento de armazenamento de muitas espécies selvagens e semidomesticadas de regiões tropicais tem sido documentada por vários autores.

$\mathrm{O}$ armazenamento de sementes permite a disponibilidade das mesmas aos programas de reflorestamento e pesquisas sobre tecnologia e fisiologia de sementes. Todavia, o sucesso do armazenamento depende do conhecimento prévio do comportamento fisiológico no armazenamento, já que sementes de diferentes espécies exigem condições especiais para a sua conservação (NERY et al., 2014).

ROBERTS (1973) classificou as sementes para fins de armazenamento em ortodoxas e recalcitrantes. As ortodoxas são descritas como relativamente pequenas, com baixas taxas de metabolismo e respiração, podendo ser dessecadas até baixos teores de umidade (2 a $5 \%$ ) e armazenadas por longos períodos de tempo em baixa temperatura $\left(-20^{\circ} \mathrm{C}\right)$, sem perda significativa da viabilidade. Já as sementes recalcitrantes são geralmente grandes, com altas taxas de metabolismo e respiração, e não toleram dessecamento a baixos conteúdos de água e nem o armazenamento a baixas temperaturas, sendo capazes de sobreviver apenas em condições especiais de armazenamento. 
Considerando a variação na sensibilidade à dessecação, ELLIS et al. (1990) sugeriram uma categoria intermediária, em que as sementes não toleram a dessecação a baixos conteúdos de água (10-12\%), mas que podem ser armazenadas a baixas temperaturas (geralmente acima de $0^{\circ} \mathrm{C}$ ). FARRANT et al. (1988) propuseram a separação das sementes recalcitrantes em "altamente", "moderadamente" e "minimamente" recalcitrantes. As sementes altamente e moderadamente recalcitrantes são sensíveis a baixas temperaturas, enquanto as minimamente recalcitrantes apresentam maior tolerância, desde que a temperatura seja superior a $0^{\circ} \mathrm{C}$.

DAWS et al. (2006) estudaram o comportamento de 104 espécies com relação à sensibilidade à dessecação e concluíram que existe uma tendência para que espécies com maior sensibilidade a dessecação apresentarem maior razão entre o envoltório da semente e a massa de matéria seca da semente, "Seed Coat Ratio" - SCR, descrito por PRITCHARD et al. (2004). Com base nesse estudo, neste trabalho foram calculados o SCR para 24 (vinte e quatro) espécies florestais visando prever a tolerância ao dessecamento das sementes pelo método SCR, bem como classifica-las quanto ao comportamento no armazenamento.

\section{MATERIAL E MÉTODOS}

Foram utilizadas sementes de 24 espécies florestais de reconhecida importância econômica regional: ipê roxo (Handroanthus impetiginosus Mart. ex D) Mattos)- Bignoniaceae, ipê amarelo (Handroanthus serratifolius A.H.Gentry S. Grose)- Bignoniaceae, amarelão (Aspidosperma vargasii A.DC.)- Apocynaceae, jatobá (Hymenaea courbaril L.)- Caesalpinoideae, castanha do Brasil (Bertholletia excelsa Bonpl.)-Lecythidaceae, timbaúba (Enterolobium schomburgkii Benth. Benth.)- Mimosoideae, jitó (Guarea sp F.Allan. ex L)- Meliaceae, jutaí (Hymenaea stignocarpa Mart. ex Hayne)- Caesalponoideae, cumarú-ferro (Dipterix odorata Aubl. wild)- Papilionoideae, mirindiba (Buchenavia grandis Ducke)- Combretaceae, breu vermelho (Tetragastris altissima Aubl.)Swart)- Burseraceae, ingá mirim (Inga. sp Mill)-Momosoideae, abiu da várzea (Pouteria reticulata Engl. Eyma)- Sapotaceae, andiroba (Carapa guianensis Aubl.)- Meliaceae, bacuri liso (Garcinia macrophylla Mart.)- Clusiaceae, pereiro (Aspidosperma macrocarpon Mart.)- Apocynaceae, tamarindo (Dialium guianense Aubl. Sandwith)- Caesalpinoideae, maçaranduba (Manilkara inundata Ducke)- Sapotaceae, sernambi de índio (Drypetes sp. Vahl)Euphorbiaceae, seringueira (Hevea brasiliensis Wild. ex A.Juss. Mull.Arg.)Euphorbiaceae, seringueirinha (Mabea anadendra Pax \& K. Hoffm.)-Euphorbiaceae, catuabinha (Abuta grandifolia Mart. Sandwith.)-Menispermeaceae, ucuúba vermelha (Virola surinamensis Rol. ex Rottb. Warb.)-Myristicaceae e caucho (Castilla ulei Warb.)-Moraceae. As sementes ou unidades de dispersão foram obtidas de frutos colhidos de uma a três matrizes selecionadas em diferentes locais do Estado do Acre, conforme Quadro 1 e Figura 1.

QUADRO 1- Localização das árvores matrizes de sementes no Estado do Acre. 


\begin{tabular}{|c|c|c|}
\hline Espécie & $\begin{array}{l}\text { Município do } \\
\text { Estado do Acre }\end{array}$ & Localização geográfica \\
\hline $\begin{array}{l}\text { Handroanthus } \\
\text { serratifolius }\end{array}$ & Sena Madureira & 0919'32,5'S/6821'38,2”W \\
\hline $\begin{array}{l}\text { Handroanthus } \\
\text { impetiginosus }\end{array}$ & Porto Acre & 0945’05”S/6740'14"'W \\
\hline Mabea anadena & Sena Madureira & 0942,8"04"S/6815'77,7”W \\
\hline Manilkara inundata & Sena Madureira & 0919'32,5'S/6821'38,2”W \\
\hline Inga. sp. & Sena Madureira & 0942'50"S/6805'48”W \\
\hline Castilla ulei & Sena Madureira & 0942,8'04”S/68익ㄱ,7”W \\
\hline Virola surinamensis & Sena Madureira & 0942,8’04”S/6815’77,7”W \\
\hline Aspidosperma vargasii & Plácido de Castro & 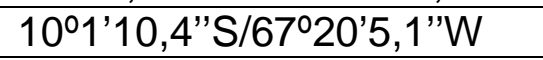 \\
\hline Abuta grandifolia & Sena Madureira & 0942,8'04"S/6815'77,7”W \\
\hline Tetragastris altissima & Plácido de Castro & 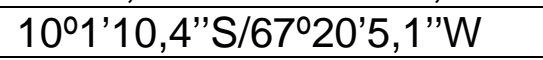 \\
\hline Guarea sp. & Plácido de Castro & 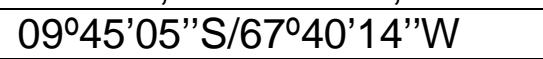 \\
\hline $\begin{array}{l}\text { Enterolobium } \\
\text { schomburgkii }\end{array}$ & Sena Madureira & 0919'32,5'S/6821'38,2”W \\
\hline Dialium guianense & Sena Madureira & 0919'32,5'S/6821'38,2”W \\
\hline Pouteria reticulata & Sena Madureira & 0943'18"S/6807'46”W \\
\hline Drypetes sp & Plácido de Castro & $10^{\circ} 1^{\prime} 10,4^{\prime \prime S} / 67^{\circ} 20^{\prime} 5,1^{\prime \prime} \mathrm{W}$ \\
\hline $\begin{array}{l}\text { Aspidosperma } \\
\text { macrocarpon }\end{array}$ & Plácido de Castro & 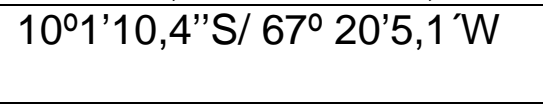 \\
\hline Garcinia macrophylla & Sena Madureira & 0943’07"S/6807'9"W \\
\hline Buchenavia grandis & Porto Acre & 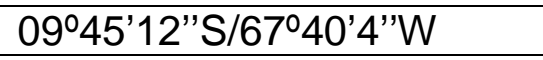 \\
\hline Hymenaea stignocarpa & Sena Madureira & 0919'32,5'S/6821'38,2”W \\
\hline Hevea brasiliensis & Porto Acre & 0945'058'S/67 $\square 40$ '3,23'”W \\
\hline Hymenaea courbaril & Porto Acre & 0945'10"S/67 40'4”W \\
\hline Bertholletia excelsa & Sena Madureira & 0919'32,5"S/6821'38,02”W \\
\hline Carapa guianensis & Sena Madureira & 0942,8’04”S/6815'77,7”W \\
\hline Dipterix odorata & Plácido de Castro & 101'10,4”S/ 6720’05,1”W \\
\hline
\end{tabular}

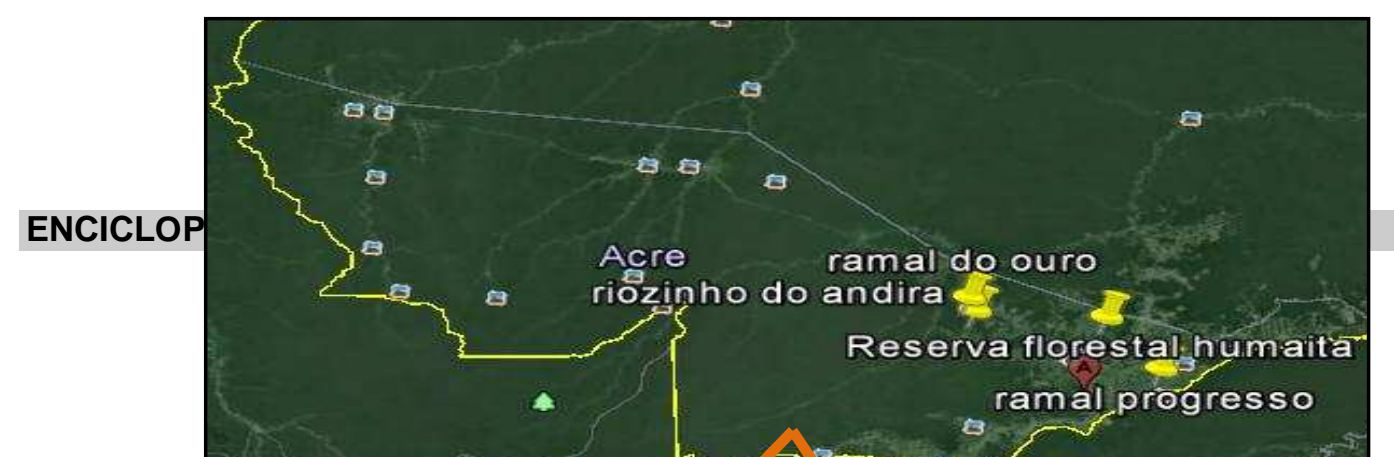


FIGURA 1: Localização das matrizes de sementes analisadas pelo método Seed Coat Ratio- SCR (Fonte: Google Earth).

Após colheita, os frutos foram imediatamente encaminhados ao Laboratório de Sementes, onde foi realizado tratamento para extração e beneficiamento das sementes. Para a previsão da tolerância ao dessecamento das sementes, foram utilizadas dez unidades de dispersão das espécies acima descritas, dissecadas em: endocarpo + testa (envoltório) e eixo embrionário + cotilédones e endosperma (semente). O material foi pesado e envolto em papel alumínio e levado à temperatura de $105^{\circ} \mathrm{C} \pm 3$, por 24 horas, com mais três pesagens subsequentes visando atingir massa estável. Posteriormente, foi calculada a massa de cada parte dessecada, utilizando balança com precisão de quatro casas decimais, sendo então calculada a relação do envoltório da semente com a massa da semente - SCR (Seed Coat Ratio - SCR): SCR= (Massa seca do envoltório (endocarpo e testa))/(Massa seca da unidade de dispersão). Para o cálculo da probabilidade da semente ser recalcitrante foi utilizada a Equação I, de acordo com DAWS et al. (2006); GOLD \& HAY (2008):

Equação I: $\mathrm{P}=\operatorname{EXP}\left(\left(3,269+(-9,974 \mathrm{SCR})+2,156^{*} \mathrm{LOG}(\mathrm{SM})\right)\right) /(1+\operatorname{EXP}((3,269+(-$ $9,974$ SCR $)+2,156^{*}$ LOG(SM))))

Sendo:

$\mathrm{SCR}=\mathrm{A} /(\mathrm{A}+\mathrm{B})$

SM ( massa seca da semente) $=A+B$

$A=$ Massa seca do embrião

$\mathrm{B}=$ Massa seca do envoltório. 
Então, se $p<0,5$ a semente é provavelmente tolerante à dessecação ortodoxa;se $\mathrm{p}>0,5$ a semente é provavelmente sensível à dessecaçãorecalcitrante.

\section{RESULTADOS E DISCUSSÃO}

A predição da classificação para fins de armazenamento com base no valor de $p$ (tolerância à dessecação), de acordo com a Equação I, para as vinte e quatro espécies amazônicas de quatorze famílias estudadas estão relacionados na Tabela 1. Foi acrescentada nesta tabela uma coluna relacionada à classificação das espécies segundo a literatura. As sementes de Handroanthus serratifolius, Handroanthus impetiginosus, Abuta grandifolia, Guarea sp. e Enterolobium scomburkii foram classificadas como ortodoxas, e as sementes de Inga sp, Pouteria reticulata, Garcinia macrophylla, Hevea brasiliensis e Carapa guianensis foram classificadas como recalcitrantes, corroborando a literatura sobre classificação de sementes para fins de armazenamento. No caso de Dialium guianensis, Aspidosperma macrocarpon, Hymenaea stignogarpa e $H$. courbaryl, consideradas pela equação I como recalcitrante, a literatura as classificam como ortodoxa. Já Manilkara inundata e Virola surinamensis, consideradas de comportamento ortodoxo, na literatura consultada foram classificadas como intermediárias. No entanto, a equação de $\mathrm{p}$ (probabilidade de recalcitrância) com base no SCR não classifica as sementes na categoria de intermediárias.

TABELA 1- Relação das espécies, sua resposta à dissecação e possibilidades de comportamento ortodoxo ou recalcitrante, baseado na massa de semente e na relação tegumento da semente (SCR) usando a equação I:

\begin{tabular}{|c|c|c|c|c|c|}
\hline Nome científico & $\begin{array}{l}\text { Massa } \\
\text { Semente } \\
(\mathrm{mg})\end{array}$ & SCR & Valor $\mathbf{P}$ & $\begin{array}{l}\text { Comportamento } \\
\text { Segundo } \\
\text { equação I }\end{array}$ & $\begin{array}{l}\text { Classificação } \\
\text { segundo } \\
\text { literatura }\end{array}$ \\
\hline $\begin{array}{l}\text { Handroanthus } \\
\text { serratifolius }\end{array}$ & 0,0216 & 0,6388 & 0,0012 & Ortodoxa & Ortodoxa $(1,2)$ \\
\hline $\begin{array}{l}\text { Handroanthus } \\
\text { impetiginosus }\end{array}$ & 0,0320 & 0,9093 & 0,0001 & Ortodoxa & Ortodoxa $(1,2)$, \\
\hline Mabea anadendra & 0,122 & 0,6344 & 0,0065 & Ortodoxa & Não encontrada \\
\hline Manilkara inundata & 0,1877 & 0,4858 & 0,0413 & Ortodoxa & $\begin{array}{l}\text { Não encontrada } \\
\text { Gênero: Intermediária } \\
\text { (1) }\end{array}$ \\
\hline Inga sp & 0,2936 & 0,0061 & 0,8869 & Recalcitrante & $\begin{array}{c}\text { Gênero Recalcitrante } \\
(1,2)\end{array}$ \\
\hline Castilla Ulei & 0,3145 & 0,9319 & 0,0008 & Ortodoxa & Não encontrada \\
\hline Virola surinamensis & 0,3217 & 0,8479 & 0,0019 & Ortodoxa & Intermediária $(1,2)$ \\
\hline $\begin{array}{l}\text { Aspidosperma } \\
\text { vargasii }\end{array}$ & 0,3452 & 0,6089 & 0,9997 & Recalcitrante & Não encontrada \\
\hline Abuta grandifolia & 0,3858 & 0,7363 & 0,0069 & Ortodoxa & $\begin{array}{c}\text { Não encontrada } \\
\text { Gênero: Ortodoxa (1) }\end{array}$ \\
\hline Tetragastris altissima & 0,5247 & 0,6906 & 0,0144 & Ortodoxa & Não encontrada \\
\hline Guarea sp & 0,5850 & 0,7492 & 0,0089 & Ortodoxa & Gênero: Ortodoxa (1) \\
\hline
\end{tabular}




\begin{tabular}{|c|c|c|c|c|c|}
\hline $\begin{array}{l}\text { Enterolobium } \\
\text { schomburgkii }\end{array}$ & 0,5937 & 0,7695 & 0,0074 & Ortodoxa & Ortodoxa (2) \\
\hline Dialium guianense & 0,6889 & 0,7395 & 0,0114 & Recalcitrante & $\begin{array}{c}\text { Não Encontrada } \\
\text { Gênero: Ortodoxa (1) }\end{array}$ \\
\hline Pouteria reticulata & 0,7878 & 0,7399 & 0,0129 & Recalcitrante & $\begin{array}{l}\text { Não encontrada } \\
\text { Gênero: Recalcitrante } \\
\text { (1) }\end{array}$ \\
\hline Drypetes sp & 0,9556 & 0,4228 & 0,2706 & Recalcitrante & Não encontrada \\
\hline $\begin{array}{l}\text { Aspidosperma } \\
\text { macrocarpon }\end{array}$ & 1,0502 & 0,8991 & 0,0034 & Recalcitrante & $\begin{array}{c}\text { Não encontrada } \\
\text { Gênero: Ortodoxa (1) }\end{array}$ \\
\hline Garcinia macrophylla & 1,1187 & 0,8276 & 0,0075 & Recalcitrante & $\begin{array}{l}\text { Não encontrada } \\
\text { Gênero: } \\
\text { Recalcitrante (1) }\end{array}$ \\
\hline Buchenavia grandis & 1,1768 & 0,0757 & 0,9349 & Recalcitrante & Não encontrada \\
\hline $\begin{array}{l}\text { Hymenaea } \\
\text { stignogarpa }\end{array}$ & 1,8996 & 0,7368 & 0,9999 & Recalcitrante & Ortodoxa (1) \\
\hline Hevea brasiliensis & 2,5135 & 0,6025 & 0,1326 & Recalcitrante & Recalcitrante $(1,2)$ \\
\hline Hymenaea courbaril & 3,3586 & 0,7597 & 0,9999 & Recalcitrante & Ortodoxa $(1,2,3)$ \\
\hline Bertholletia excelsa & 5,442 & 0,5377 & 0,3756 & Recalcitrante & Não encontrada \\
\hline Carapa guianensis & 6,0069 & 0,7800 & 0,0556 & Recalcitrante & Recalcitrante $(1,2)$ \\
\hline Dipterix odorata & 10,477 & 0,1025 & 0,9984 & Recalcitrante & Não encontrada \\
\hline
\end{tabular}

${ }^{1}$ Kew Garden - Millennium Seed Bank

2 Lima et al. (2014)

${ }^{3}$ CARVALHO et al. (2006)

Durante o processo de germinação, sementes ortodoxas perdem gradualmente a tolerância à dessecação. Quando ocorre a protusão radicular, a nova plântula é sensível à dessecação, e pode sofrer danos irreversíveis em caso de escassez de água. A radícula é a primeira estrutura do vegetal a perder a tolerância à dessecação, o que ocorre logo após a sua protrusão (RODRIGUES et al., 2015).

Durante o desenvolvimento da semente algumas proteínas e açúcares específicos são sintetizados de forma tardia que podem estar associados à capacidade de tolerância à dessecação. Entre elas estão as proteínas LEA (Late embryogenesis abundant), que tem a função na adaptação e na proteção das células contra a desidratação. O desenvolvimento de sementes de espécies ortodoxas é dividido em três fases: histo-diferenciação, maturação e secagem. Durante a fase de maturação, as sementes passam a adquirir a tolerância à dessecação. Iniciado o processo de secagem, estas podem atingir de $5 \%$ a $15 \%$ de umidade, entrando então no estado de quiescência, o que causa redução no seu metabolismo, permitindo que as sementes permaneçam vivas mesmo em condições adversas. Ao serem reidratadas e em condições favoráveis, reativam o metabolismo, iniciando então o processo germinativo (PELIISARI et al, 2013).

$\mathrm{Na}$ Figura 2 estão apresentadas as espécies estudadas com relação à massa da semente e relação tegumento/semente (SCR). Verifica-se que algumas espécies apresentaram-se com distribuição próxima à linha correspondente à probabilidade de sensibilidade à dessecação, embora segundo a Equação I foram classificadas como ortodoxas ou recalcitrantes. Segundo a literatura consultada, estas espécies foram classificadas em intermediarias ou não foram classificadas quanto ao armazenamento (Tabela 1). 


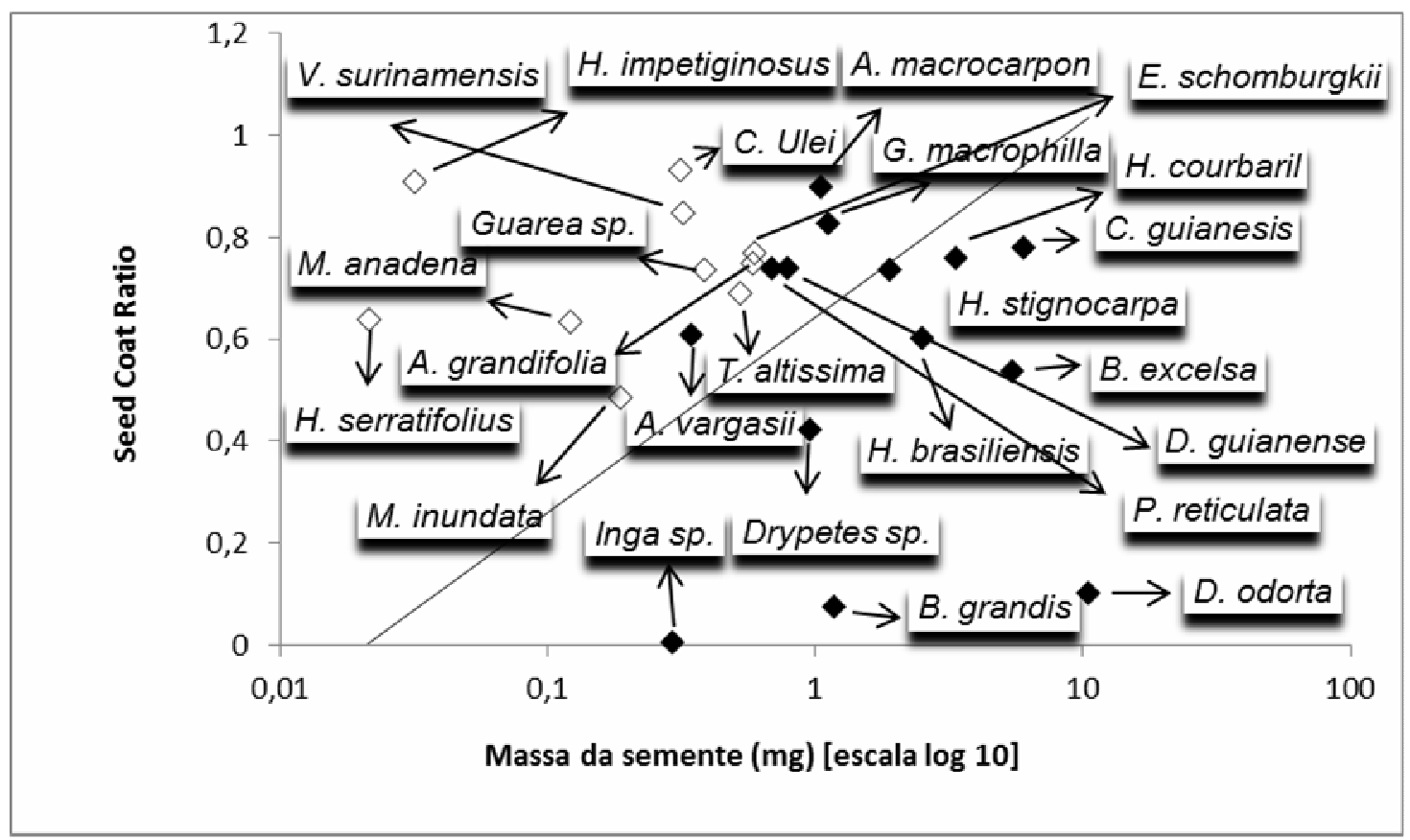

FIGURA 2- Efeito da Massa de Semente e Relação tegumento-semente SCR na tolerância à dessecação de 24 espécies amazônicas. Símbolos abertos correspondem a espécies com sementes tolerantes à dissecação (ortodoxas) e símbolos fechados correspondem a espécies sensíveis à dessecação (recalcitrantes). A linha corresponde à probabilidade a sensibilidade a dessecação, baseada na Equação I. A linha corresponde à probabilidade de sensibilidade à dessecação de 0,5 , isto é, o ponto no qual, com base na análise de regressão (Equação I), as sementes são igualmente susceptíveis de serem sensíveis ou tolerantes à dessecação (Adaptado de DAWS et al., 2006).

Segundo REGO et al. (2013), existem indicações que o teor mínimo de água na semente que pode levar à perda da viabilidade de sementes recalcitrantes é altamente variável dentro e entre as espécies. Assim, a sensibilidade à dessecação parece variar de extremamente a moderadamente, quando a taxa de secagem das sementes pode ser um dos fatores que contribuem para a variação na sensibilidade.

No entanto, alguns estudos realizados sobre armazenamento de sementes florestais recalcitrantes citam diversas metodologias para manter a viabilidade das mesmas por algum período de tempo. Para sementes de Tabebuia impetiginosa a conservação é favorecida pela manutenção com teores de água entre 4,2 e $12,5 \%$ a $-10^{\circ} \mathrm{C}$ e entre 4,2 e $8,4 \%$ a $20{ }^{\circ} \mathrm{C}$ (MARTINS et al. 2 012). Para sementes recalcitrantes de Inga vera (BONJOVANI \& BARBEDO, 2014), a incubação de embriões em uma solução PEG a -2,0 MPa aumenta sua tolerância à dessecação. Já Bauhinia forficata possui capacidade de re-indução de tolerância à dessecação com até $4 \mathrm{~mm}$ de comprimento de radícula, quando tratada com solução de PEG à 1,4 Mpa (RODRIGUES et al, 2015). 
Para sementes de café (Coffea arabica), a secagem lenta a sombra até $20 \%$ de teor de água propicia a conservação da qualidade em sementes de cafeeiro por até doze meses de armazenamento (ABREU et al., 2014). No caso de Campomanesia. damantium, as sementes são sensíveis à dessecação a uma redução do teor de umidade a 21,1 \% (DRESCH et al., 2015). Sementes de Caesalpinia echinata toleram a secagem até um teor de água de $5 \%$ (base úmida) em soluções salinas $\left(\mathrm{CaCl}_{2}\right.$ e $\left.\mathrm{ZnCl}_{2}\right)$ sendo que, a maioria das sementes permanecem viáveis, mas mantêm a viabilidade por curtos períodos, quando em temperatura ambiente (MARTINI NETO \& BARBEDO, 2015).

Em condições de secagem lenta, as sementes de Blepharocalyx salicifolius e Casearia decandra foram sensíveis à redução de umidade entre $29 \%$ e $25 \%$ e perderam a viabilidade em torno de $14 \%$ e $8 \%$ de umidade, respectivamente (REGO et al., 2013). Para sementes de Eugenia pyriformis, a tolerância à dessecação depende do estádio de maturidade fisiológica no momento da dispersão, que por sua vez depende das condições térmicas e hídricas do ambiente durante a sua formação (LAMARCA et al., 2016).

Segundo COSTA et al. (2016), um método prático para aumentar a longevidade de sementes recalcitrantes pode ser o armazenamento de sementes germinadas, em que a tolerância à dessecação pode ser re-induzida por um tratamento osmótico e/ou com ácido abscísico. Sementes germinadas de Sesbania virgata com radícula de $1 \mathrm{~mm}$ de comprimento toleram dessecação e armazenamento por até três meses sem redução significativa na longevidade. $O$ tratamento osmótico melhora a tolerância à dessecação nessas sementes, mas não a longevidade.

\section{CONCLUSÕES}

A previsão da tolerância da semente ao dessecamento pelo método "Seed Coat Ratio"- SCR foi eficiente para $42 \%$ das espécies estudadas. Do restante, $33 \%$ não foi encontrada na literatura e $6 \%$ apresentaram resultados opostos ou foram classificadas como intermediária. Assim, sugere-se que metodologia, embora rápida e prática, deve ser utilizada associando-se outros critérios na pré-classificação das sementes, uma vez que o método apenas classifica as sementes em ortodoxas e recalcitrantes, não considerando a categoria intermediária ou o nível de recalcitrância das sementes.

Além disso, ainda existem lacunas no conhecimento científico quanto à classificação para fins de armazenamento para a maioria das espécies florestais amazônicas.

\section{AGRADECIMENTOS}

Ao $C N P Q$, pelo apoio à realização da pesquisa através do projeto CT Amazônia, coordenado pela Dra. Isolde Ferraz- do Instituto Nacional de Pesquisas da Amazônia INPA/AM; Ao Dr. Evandro José Linhares Ferreira, pesquisador do INPA/AC em convenio com a Universidade Federal do Acre- UFAC, pelo apoio na elaboração do abstract; e à equipe do Laboratório de Sementes do Parque Zoobotânico da UFAC- João Lopes Firmino, Pedro de Albuquerque Ferraz, Plinio Carlos Mitoso e Francisco Félix Amaral, pela colheita de sementes e auxilio na montagem e acompanhamento da pesquisa. 


\section{REFERÊNCIAS}

ABREU, L. A. S.; VEIGA, A. D.; PINHO, E.V. R. V.; MONTEIRO, F. F. ROSA, S. D. $V$. F. Behavior of coffee seeds to desiccation tolerance and storage. Journal of Seed Science, v.36, n.4, p.399-406, 2014.

BONJOVANI, M. R. ; BARBEDO, C. J. Induction of tolerance to desiccation and to subzero temperatures in embryos of recalcitrant seeds of inga. Journal of Seed Science, v.36, n.4, p.419-426, 2014.

BRASIL. Ministério da Agricultura, Pesca e Abastecimento. Regras para análise de sementes. Brasília: Secretaria Nacional de Defesa Agropecuária; 2009.

http://www.agricultura.gov.br/arq_editor/file/2946_regras_analise_sementes.pdf

CARVALHO,L. R.; SILVA, E. A. A., DAVID, A. C. Classificação de sementes florestais quanto ao comportamento no armazenamento. Revista Brasileira de Sementes, v. 28, n. 2, p.15-25, 2006.

COSTA, M. C. D.; FARIA, J. M. R., JOSÉ, A. C., LIGTERINK, W., HILHORST, H. W.M. Desiccation tolerance and longevity of germinated Sesbania virgata (Cav.) Pers. Seeds. Journal of Seed Science, v.38, n.1, p50-56, 2016.

DAWS, M. I.; GARWOOD, N. C.; PRITCHARD, H. W. Prediction of Desiccation Sensitivity in Seeds of Woody Species: A Probabilistic Model Based on Two Seed Traits and 104 Species. Annals of Botany v.97, p. 667-674, 2006.

DRESCH, D. M.; MASETTO, T.E.; SCALON, S. P.Q. Campomanesia adamantium (Cambess.) O. Berg seed desiccation: influence on vigor and nucleic acids. Anais da Academia Brasileira de Ciências, v.87, n.4, p. 2217-2228, 2015.

ELLIS, R.H.; HONG, T.D.; ROBERTS, H. An intermediate category of seed storage behavior? I. Coffee. Journal of Experimental Botany v.41, n.230, p. 1167-1174, 1990.

FARRANT, J.M.; PAMMENTER, N.W.; BERJAK, P. Recalcitrance: a current assessment. Seed Science and Technology, v.16, n.1, p. 155-166, 1988.

GOMES, J. P.; OLIVEIRA, L.M.; SALDANHA, A. P.; MANFREDI, S., FERREIRA, P. I. Secagem e Classificação de Sementes de Acca sellowiana (O. Berg) Burret Myrtaceae quanto à Tolerância à Dessecação e ao Armazenamento. Floresta e Ambiente, v.20, n.2, p. 207-215, 2013.

GOLD, K., HAY, F. Identifying desiccation- sensitive seeds. Millennium Seed Bank Project, Wakehurst Place, Ardingly, Technical Information Sheet 10, 2008.

HONG TD, LININGTON S, ELLIS RH. Seed storage behaviour: a compendium. Rome: International Plant Genetic Resources Institute; 1996. 
LAMARCA, E. V.; CAMARGO, M. B. P.; TEIXEIRA, S. P.; SILVA, E. A. A., FARIA, J. M. R.; BARBEDO, C. J. Variations in desiccation tolerance in seeds of Eugenia pyriformis: dispersal at different stages of maturation. Revista Ciência Agronômica, v. 47 , n. 1, p. 118-126, 2016.

LIMA JR, M.; HONG, T.D., ARRUDA, Y.M.B.C., MENDES, A.M.S; ELLIS, R.H. Classification of seed storage behaviour of 67 Amazonian tree Species. Seed Sci. \& Technol., $\quad$ v.42, p. 363-392, 2014. Disponível em: <http://doi.org/10.15258/sst.2014.42.3.06>. doi: 10.15258/sst.2014.42.3.06

MARTINI NETO, N.; BARBEDO, C. J. Viability of Brazilwood seeds (Caesalpinia echinata Lam.) stored at room temperature in controlled atmospheres. Journal of Seed Science, v.37, n.2, p. 93-101, 2015.

MARTINS, L., LAGO, A. A., ANDRADE, A. C. S. Teor de água, temperatura do ambiente e conservação de sementes de ipê-roxo. Revista Árvore v,36, n.2, p.203$210,2012$.

NERY, M. C.; DAVIDE, A. C.; SILVA, E. A. A.; SOARES, G. C. M., NERY, F.C. Classificação fisiológica de sementes florestais quanto a tolerância à dessecação e ao armazenamento. CERNE v. 20, n. 3 p. 477-483, 2014.

PELISSARI, F.; SILVA, C. J.; VIEIRA C. V. Classificação Quanto a Tolerância à Dessecação e ao Armazenamento de Sementes de Cassia fistula L. Scientific Electronic Archives, v. 2, p. 1-5, 2013.

PRITCHARD, H.W.; DAWS, M.I.; FLETCHER, B.J.; GAMÉNÉ, C. S.; MSANGA, H.P.; OMONDI, W. 2004. Ecological correlates of seed desiccation tolerance in tropical African drayland trees. American Journal of Botany v.91, p. 863-870.

REGO, S. S.; NOGUEIRA, A. C. , MEDEIROS, A. C. S. PETKOWICZ, C. L. O.; SANTOS, A. F. Physiological behaviour of Blepharocalyx salicifolius and Casearia decandra seeds on the tolerance to dehydration. Journal of Seed Science, v.35, n.3, p.323-330, 2013.

ROBERTS, E.H. Predicting the storage life of seeds. Seed Science and Technology v.1, n.3, p. 499-514, 1973.

RodRigues, A. C.; AlvarengA, A. A., RiBeiRO, D. E.; GUIMARÃES, R. M.; ALVES, E.; SILVA JUNIOR, J. M. Reindução da tolerância à dessecação em sementes de Bauhinia forficata LINK (Fabaceae). CERNE, v. 21 n. 4, p. 579-586, 2015.

VINAYACHANDRA; CHANDRASHEKAR, K. R. Seed storage behavior of Knema attenuata, an endemic species of Western Ghats, India. Journal of Forestry Research, v.2, n.4, p. 611-614, 2011. 